\title{
A COMPACT X-RAY LITHOGRAPHY LATTICE USING SUPERFERRIC MAGNETS
}

\author{
Charles A. Swenson, F. R. Huson, and W. W. Mackay \\ Department of Physics, Texas A\&M University, College Station, Texas 77843 \\ Texas Accelerator Center, The Woodlands, Texas 77381 \\ L.K. Chen and S. Ohnuma \\ Department of Physics, University of Houston, Houston, Texas 77204-5504 \\ Texas Accelerator Center, The Woodlands, Texas 77381
}

\begin{abstract}
A conceptual lattice design for a very compact superconducting synchrotron dedicated to $x$-ray lithography is presented. The synchrotron radiation produced in the high field superconducting magnets has a critical wavelength of $10 \mathrm{~A}$ at a beam energy of about $787 \mathrm{MeV}$. The size and angular divergence of the beam in this lattice can satisfy the future requirements for x-ray lithography: $\sigma_{x, y} \leq 1 \mathrm{~mm}$, and $\sigma_{x, y}^{\prime} \leq 1 \mathrm{mr}$. The paper will present an optimization of the lithography parameters $\sigma_{x}$, and $\sigma_{x}^{\prime}$ for this lattice configuration.
\end{abstract}

\section{Introduction}

The requirements for the industrial application of $x$-ray lithography pose a unique set of constraints upon the design of an electron synchrotron. First and most fundamentally the optical source properties of the beam must satisfy the lithography requirements: $\sigma_{x, y} \leq 1 \mathrm{~mm}$, and $\sigma_{x, y}^{\prime} \leq 1 \mathrm{mr}$. Additionally the radiation spectrum should be adjusted so that the majority of the radiation bandwidth falls between 6 and $10 \AA$. These physical requirements have been easily satisfied by existing research machines. ${ }^{2,3}$

A large synchrotron with many components is not readily incorporated into semiconductor manufacturing systems. Traditional synchrotrons are too large, and have an excessive number of components. Such facilities do not represent viable industrial machines because the system cost and reliability are directly related to the machine's size and the number of components used. Additionally this type of production facility is unmanageable because key responsibilities must be delegated to several people. Any single failure can stop the production from all the $x$-ray mask exposers associated with the ring. Clearly if the synchrotron light source is to become useful for industrial $x$ ray lithography, the operational requirements of the light source must be tailored to the requirements of the production line. The ultimate machine design must emphasize compactness, reliability, and technical simplicity. The design philosophy advocated in this paper is as follows: 1.) Simplify the light source dipole magnet design by removing the radial field gradient. 2.) Select the largest dipole field strengths where ferric magnets can be constructed efficiently, 3.) Determine the minimal lattice configuration where the optical source properties for lithography can be satisfied. 4.) Design a compact intermediate energy injection system to avoid the problems associated with low energy injection.

\section{Review of Existing Machine Concepts}

\section{Single Gradient Machines}

The single gradient or cylindrically symmetric machine designs have been thoroughly characterized for lithography in previous papers. ${ }^{1,4}$ If the optical source properties of the beam $\sigma_{x}$ and $\sigma_{z}^{\prime}$, and the critical wavelength of the synchrotron radiation $\lambda_{c}$ are treated as constraints for lithography, the magnetic field is specified. When these lithography constraints are applied $\sigma_{x}$

Work supported in part by the U.S. Department of Energy. and $\sigma_{x}^{\prime}$ are analytically defined in terms of the field index $n, \lambda_{c}$, and $B$. Both the energy and the radius of the beam become scaled by the magnetic field $B$ when $\lambda_{\mathrm{c}}$ is fixed. It is instructive to plot the contours of $\sigma_{x}$ and $\sigma_{x}^{\prime}$ in the plane defined by $B$, and the field index $n$ for a particular choice of $\lambda_{c}$. The particular functional relations are:

$$
\begin{gathered}
B=B\left(n, \lambda_{c}, \sigma_{x}\right)=\left(\frac{19.83}{\sigma_{x}} \lambda_{c}^{-\frac{3}{4}}\left(\frac{3}{n(3-4 n)}\right)^{\frac{1}{2}}\right)^{\frac{1}{s}} \\
B=B\left(n, \lambda_{c}, \sigma_{x}^{\prime}\right)=\lambda_{c} n^{2}\left(\frac{\sigma_{x}^{\prime}}{1.38}\right)^{4}
\end{gathered}
$$

where $\lambda_{c}$ is in angstroms, $B$ in tesla, $\sigma_{x}$ in mm, and $\sigma_{x}^{\prime}$ is in mr. Figure 1 shows such a plot for $\lambda_{c}-10$ angstroms. Note that the curves for $\sigma_{x}=1 \mathrm{~mm}$ and $\sigma_{x}^{\prime}=1 \mathrm{mr}$ do not intersect. There is no single gradient machine configuration which can satisfy the lithographer's objective of $\sigma_{x} \leq 1 \mathrm{~mm}$, and $\sigma_{x}^{\prime} \leq 1 \mathrm{mr}$.

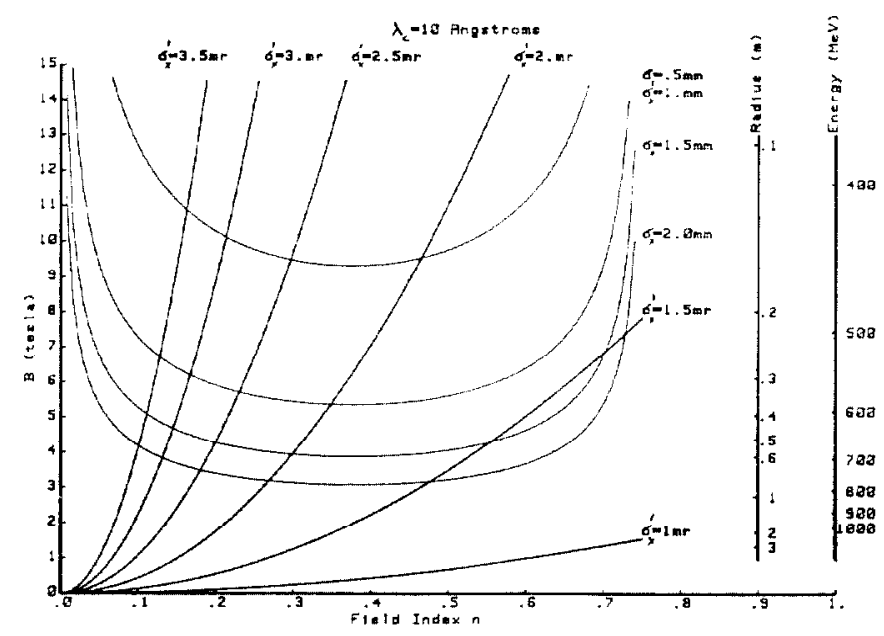

Figure 1: Contour plots of $\sigma_{\mathbf{r}}$ and $\sigma_{t}^{\prime}$ in the $B, n$ plane for $\lambda_{c}=10 A$ in single gradient machines. Both $\rho$ and $E$ scale with the magnetic field $B$.

\section{Racetrack Structures}

Racetrack designs use two $180^{\circ}$ combined function dipole magnets. The presence of the radial field gradient serves to reduce the natural horizontal emittance of the beam. This can bc seen by an analysis of the synchrotron integrals and their relation to the natural emittance. The common practice in designing a compact racetrack synchrotron for lithography is to use a gradient in the dipoles to reduce the natural emittance of the beam. This is how a beam with an acceptable size and divergence is achieved in such compact machines. Combined function dipoles are required in these machincs for symmetric and asymmetric lattice configurations. It is crucial that the high-field dipoles be made with a precisely controlled radial gradient because this is the only means which allows $180^{\circ}$ racetrack machines to be practical for lithography. 


\section{Symmetric Cell Analysis}

The following working assumptions were made in the analysis of the optics for a lithography source. 1.) There is always one dipole magnet per lattice cell. 2.) The linear lattice functions are mirror symmetric about the center of the dipole. In such lattices the center of the dipole magnet is a symmetry axis where the lattice functions can be specified in terms of the light source parameters desired. All the essential lattice functions can be evaluated after this specification. The linear machine functions are

$$
\begin{gathered}
\eta(s)=\eta_{0} \cos \left(\sqrt{1-n} \frac{s}{\rho}\right)+\frac{\rho}{1-n}\left(1-\cos \left(\sqrt{1-n} \frac{s}{\rho}\right)\right), \\
\eta^{\prime}(s)=\left(\frac{1}{\sqrt{1-n}}-\frac{\eta_{0}}{\rho} \sqrt{1-n}\right) \sin \left(\sqrt{1-n \frac{s}{\rho}}\right), \\
\beta(s)=\beta_{0} \cos ^{2}\left(\sqrt{1-n} \frac{s}{\rho}\right)+\frac{\rho^{2}}{\beta_{0}} \sin ^{2}\left(\sqrt{1-n} \frac{s}{\rho}\right), \\
\beta^{\prime}(s)=\frac{s}{2} \sqrt{1-n}\left(\frac{\rho}{\beta_{0}}-\frac{\beta_{0}}{\rho}\right) \sin \left(2 \sqrt{1-n} \frac{s}{\rho}\right) .
\end{gathered}
$$

With this formalism the synchrotron integrals need only be evaluated over half of a magnet. The partial integrals may then be multiplied by $2 N$, to yield the entire lattice integrals, where $N$, is the lattice periodicity of the machine being designed. All the synchrotron integrals, the emittance, energy spread, and beam parameters in the magnets are functions of the magnetic radius $\rho$, the magnet bend angle $\theta_{m}$, the magnetic field index $n$, and the heam energy. Sperifically: $:^{5,6}$

$$
\begin{gathered}
I_{j}=f\left(\beta_{0}, \eta_{0}, n, \rho, \theta_{m}\right) ; j=1 \text { to } 5 \\
\left(\frac{\sigma_{E}}{E}\right)^{2}=C_{q} \gamma^{2}\left(\frac{I_{3}}{2 I_{2}+I_{4}}\right) \\
\epsilon=C_{q} \gamma^{2}\left(\frac{I_{5}}{I_{2}-I_{4}}\right) \\
\sigma_{x}=\left(\beta(s) \epsilon+\left(\frac{\sigma_{E}}{E}\right)^{2} \eta^{2}\right)^{\frac{1}{2}} \\
\sigma_{*}^{\prime}=\left(\frac{1+\alpha^{2}(s)}{\beta(s)} \epsilon+\left(\frac{\sigma_{E}}{E}\right)^{2} \eta^{\prime 2}\right)^{\frac{1}{2}},
\end{gathered}
$$

where $\gamma$ is the Lorentz factor.

The optical source properties of the beam are now determined in terms of three classes of variables: the bend angle $\theta_{m}$; the constraints, $\gamma$ and $\rho$; and the variables $\beta_{0}, \eta_{\mu}$. and 11 . $1 \psi_{\mu}$ matching functions are varied to satisfy the requirements for lithography, given a single cell mirror symmetric lattice defined by $\theta_{m}$. This analysis is valid for rectangular magnets as well as sector magnets because the integrals are evaluated from the symmetry point at the magnet center outward along the design orbit. In the analysis we plot $\sigma_{x}$ and $\sigma_{x}^{\prime}$ at the mirror symmetry point of the dipoles versus $\beta_{0}$ for varions values of the dispersion function $\eta_{0}$. Figure $2 \mathrm{a}$ is a plot for a $180^{\circ}$ bend $3 \mathrm{~T}$ dipole. Figure $2 \mathrm{~b}$ represents a $90^{\circ} 3 \mathrm{~T}$ dipole. In both cases the value of the dispersion function is $\eta_{0}=.25$ meters. The curves for $\sigma_{x}$ and $\sigma_{x}^{\prime}$ do not fall far below the $1 \mathrm{~mm}$ or $1 \mathrm{mr}$ values for lithography in $180^{\circ}$ or $120^{\circ}$ magnets. The $90^{\circ}$ zero gradient magnets are suitable for lithography over a wide range of matching variables. Figures $2 \mathrm{a}$ and $2 \mathrm{~b}$ also plot the curves for variable field index $n$ in the dipoles. Notice that a beam suitable for lithography can be produced using a $180^{\circ} 3 \mathrm{~T}$ bend with a radial gradient. The presence of the gradient reduces the natural emittance of the beam, thereby reducing the beam size and divergence.
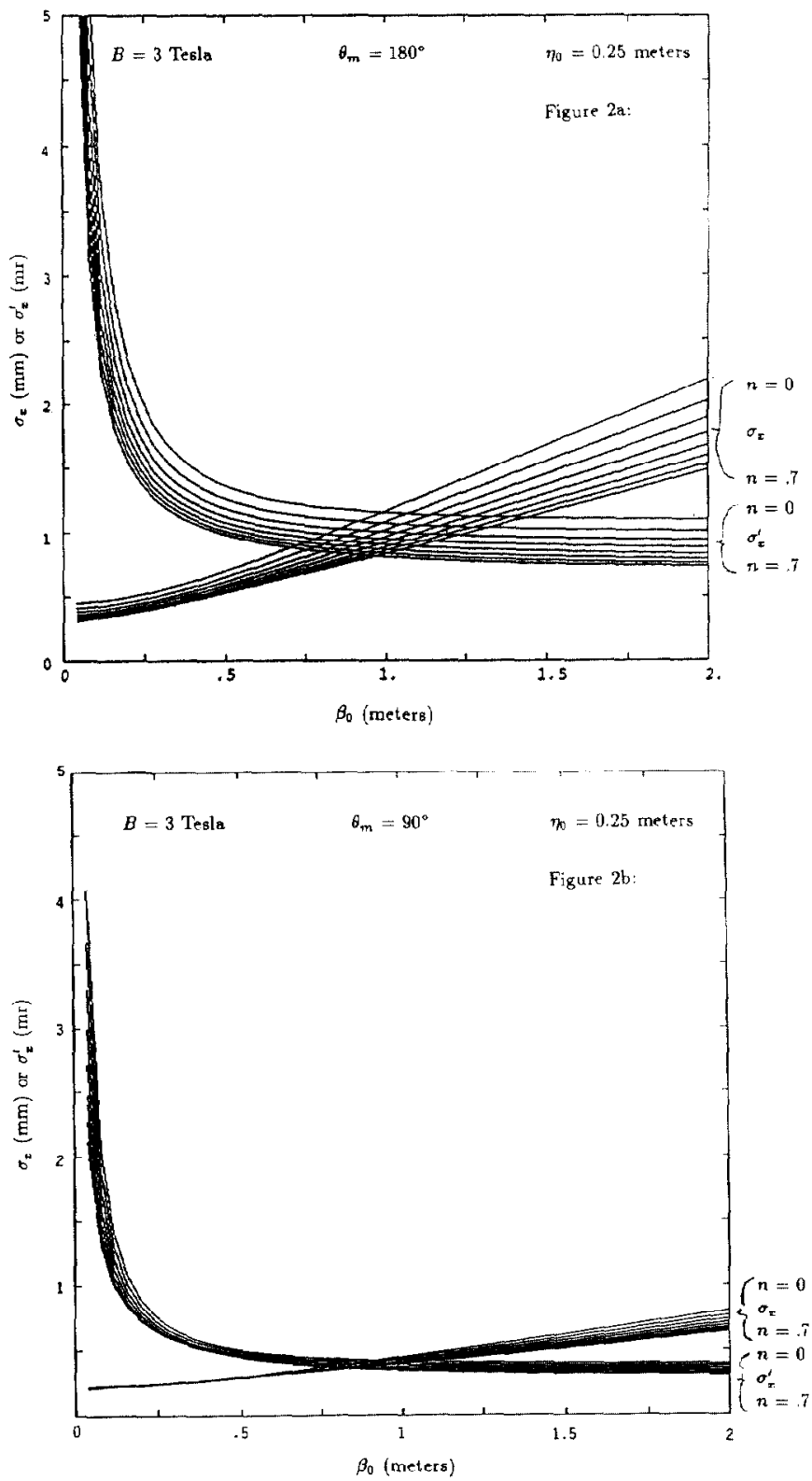

Figure 2: Plots of $\sigma_{x}$ and $\sigma_{x}^{\prime}$ vs. $\beta_{0}$ at the dipole mirror symmetry point. a.) Plot for a $180^{\circ} 3 \mathrm{~T}$ dipole with $\eta_{0}=25 \mathrm{~m}$. b.) Pint fnr a $90^{\circ} 3 \mathrm{~T}$ dipole with $\eta_{0}=.25 \mathrm{~m}$

\section{Light Source Lattice}

\section{Dipole Magnet Selection}

A $3 \mathrm{~T}$ zero gradient superferric dipole has been selected as the design magnet. Such magnets are efficient and elegant in their construction. The use of iron in conjunction with small flexible superconducting wires allows the edge field effects to be minimized. Magnet construction is further simplified by the exclusion of the radial field gradient. Three tesla is the chosen magnetic field intensity because $\mathrm{C}$ magnets lose their efficiency above this value.

\section{Lattice Cell Structure}

The light source layout and linear lattice functions are displayed in figure 3 . Each lattice cell has a mirror symmetry about the center of the dipole magnet. There are three quadrupoles in each straight section. The total length of each straight section including the quadrupoles is 3.85 meters. The symmetric lattice cell begins and ends in the central quadrupole. This asymmetric quadrupole configuration allows the lattice functions to be matched for lithography while optimizing a stable operating 
point in tune space. The optical source properties of the beam satisfy the requirements for lithography throughout the dipole magnets.

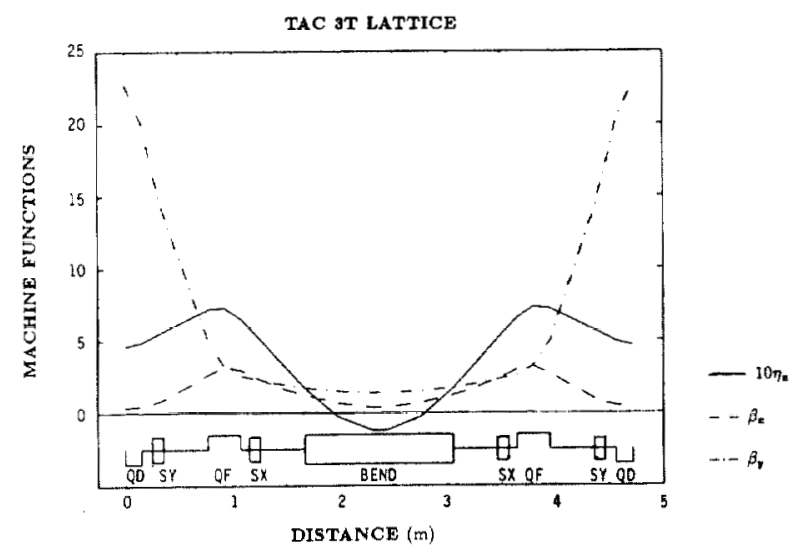

Figure 3: Linear lattice functions for a single lattice cell.

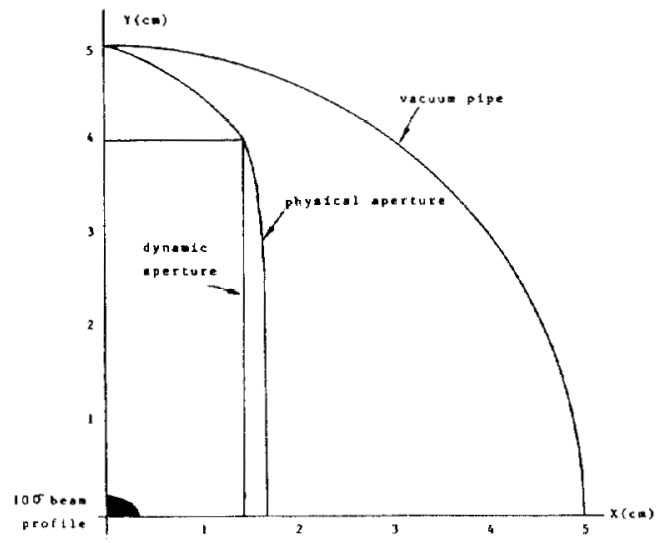

Figure 4: Dynamic aperture. Particles were sracked with $\frac{\delta E}{E}=10 \sigma_{E}$ for 40000 turns. The "physical aperture" is an analytic estimate of the aperture in the absence of nonlinearities and coupling.

\section{Preliminary Tracking Results}

Initial tracking simulations with sextupoles for correction of chromaticity have been made using the second order code DIMAD. ${ }^{7}$ Particles have been tracked successfully for one damping time within an aperture of $15 \mathrm{~mm}$ for $\delta E=10 \sigma_{E}$. See figure 4. A detailed analysis of the edge effects has not yet been completcd.

\section{Injector Design Concept}

The injection system consists of a $30 \mathrm{MeV}$ microtron which injects a 15 ma pulse of beam current into a compact 30-300 $\mathrm{MeV}$ booster synchrotron. Single turn injection is used to accumulate beam in the booster. The booster cycle rate is $1 \mathrm{~Hz}$. The ideal rate of current accumulation in the storage ring should be between 2 and 6 ma per cycle. A more detailed presentation of the booster is presented in another paper in these proceedings. Intermediate energy injection, $300 \mathrm{MeV}$, has been adopted to simplify the accumulation of current in the storage ring. At 300 $\mathrm{MeV}$ the damping times for the light source are $\tau_{x}=45.0 \mathrm{~ms}$, $\tau_{y}=46.1 \mathrm{~ms}, \tau_{\epsilon}=23.4 \mathrm{~ms}$ This corresponds to roughly ten damping times between injection cycles.

The injector system has been selected as a compromise that provides an intermediate energy beam within a reasonably compact size. The booster size is roughly two times that required for a single $100 \mathrm{MeV}$ racetrack microtron. This small increase in size for the total injector system is minimal, if the booster is used to service several storage rings for lithography.

\section{Table 1 - Linear Lattice Parameters}

\begin{tabular}{|c|c|c|}
\hline \multicolumn{2}{|l|}{ Beam Energy $(\mathrm{MeV})$} & 787 \\
\hline \multicolumn{2}{|l|}{ Number of Superperiods } & 4 \\
\hline \multicolumn{2}{|c|}{ Circumference (meters) } & 18.88 \\
\hline \multicolumn{2}{|c|}{ Number of Bending Magnets } & 4 \\
\hline \multicolumn{2}{|l|}{ Magnetic radius $(\mathrm{cm})$} & 87.44 \\
\hline \multicolumn{2}{|l|}{ Peak Magnetic Field (T) } & 3.0 \\
\hline \multicolumn{2}{|l|}{ Critical Wavelength $(\dot{A})$} & 10.0 \\
\hline \multicolumn{2}{|c|}{ Energy Loss per Turn (keV) } & 38.8 \\
\hline \multicolumn{2}{|c|}{ Horizontal Betatron Tune } & 3.23 \\
\hline \multicolumn{2}{|l|}{ Vertical Betatron Tune } & 1.16 \\
\hline \multicolumn{2}{|c|}{ Momentum Compaction Factor } & $-.724 \times 10^{-}$ \\
\hline \multicolumn{2}{|c|}{ Horizontal Natural Chromaticity } & -3.1 \\
\hline \multicolumn{2}{|c|}{ Vertical Natural Chromaticity } & -8.3 \\
\hline \multicolumn{2}{|c|}{ Natural Beam Emittance (meter-rad) } & $2.03 \times 10^{-7}$ \\
\hline \multicolumn{2}{|c|}{ Natural Energy Spread $\left(\frac{\sigma_{E}}{E}\right)$} & $0.725 \times 10^{-3}$ \\
\hline \multirow[t]{3}{*}{ Damping Times (ms): } & $\tau_{x}$ & 2.49 \\
\hline & $T_{y}$ & 2.56 \\
\hline & $\tau_{\epsilon}$ & 1.29 \\
\hline \multicolumn{2}{|l|}{ Number of Quadrupoles } & 12 \\
\hline \multicolumn{2}{|c|}{ Quadrupole Length (meters) } & 0.3 \\
\hline \multicolumn{2}{|l|}{ RF Frequency (MHz) } & 476 \\
\hline \multicolumn{2}{|l|}{ Harmonic Number } & 30 \\
\hline
\end{tabular}

\section{Conclusion}

We present a method for optimizing compact light source lattices with mirror symmetric cells containing a single dipole in each superperiod. Zero gradient magnets berome practical for lithography when the magnet bend angle is $90^{\circ}$ or less. For an industrial lithography light source all components of a machine must be reliable and designed with sound engineering principals. The 3T zero gradient superconducting dipoles represent a high field design goal which is both achievable and applicable to mass production. A compact stable lattice design using these magnets has been presented. The machine footprint falls within a $5 \times 5$ square meter area. This machine can be fabricated in two $2.5 \times 5$ square meter sections for transportation and remote assembly. The light source design uses a compact $300 \mathrm{MeV}$ injector which is discussed elsewhere in these conference proceedings.

\section{References}

1. N. Takahashi, "Compact Superconducting SR Ring for X-ray Lithography," Nucl. Inst. and Meth. B24/25, 425 (1987).

2. Proceedings of the Workshop on Compact Storage Ring Technology: Applications to Lithography, BNI 52005 (1986).

3. Report of the Second Workshop on Synchrotron Radiation Sources for X-ray Lithography, BNL 38789 (1986).

4. G. A. Decker and B. C. Craft, "Evaluation of Compact Synchrotron Optics for X-ray Lithography", 1987 IEEE Particle Accelerator Conference, IEEE No. 87CH2387-9, (1987).

5. R. H. Helm, M. J. Lee, P. L. Morton, and M. Sands, "Evaluation of Synchrotron Radiation Integrals", IEEE Trans. Nucl. Sci. NS-20, No. 4, 900 (1973).

6. M. Sands, "The Physics of Electron Storage Rings", SLAC-121, UC 28 (ACC) (1970).

T. R. V. Severanckx, and K. L. Brown, "Users Guide to the Program DIMAD", SLAC-285, IC-28 (A) (1985). 